\title{
Neuropathological Changes and Abnormal RT97 Staining of Neurofilaments in the Host Hippocampus, One Year After Intrahippocampal Transplantation
}

\author{
S.N. Patel, T.R. Kershaw and J.D. Sinden \\ The Institute of Psychiatry, Departments of Psychology and Neuropathology, \\ DeCrespigny Park, London SE5 8AF, UK
}

We report here several neuropathological alterations in the host brain one year after the placement of intrahippocampal neural allografts of cholinergic-rich basal forebrain tissue in fimbria-fornix lesioned rats. The immunological reaction against the allografts had resulted in damage to the host tissue surrounding the graft, specifically to the host cerebral cortex and the corpus callosum. In the host hippocampus, there was damage to the CA1 cell layer and abnormal neurofilament accumulations in perikarya and in axonal swellings were revealed by RT97 immunohistochemistry. These changes were not found within the grafts, which appeared relatively healthy.

F344 rats received fimbria-fornix lesions and PVG foetal basal forebrain tissue into the host hippocampus (bilateral). These rats also received injections of irradiated PVG splenocytes (s.c.) 10-11 mo. later, in an attempt to cause an immunological rejection of the PVG neural grafts. The grafts were examined immunohistochemically 12 mo. post-grafting with various markers (results presented previously /5/. This abstract concerns labelling with an RT97 antibody.

Fibres, cell bodies and axonal swellings were stained with the RT97 antibody in the host hippocampi of the transplanted rats. Numerous stained cell bodies and axonal swellings were found almost everywhere within the hippocampus of one particular transplanted rat, but only 2-3 were observed in the thalamus and cortex and no such abnormal structures were observed in the transplants themselves. Of the 9 (out of 12) transplanted rats examined with RT97 staining all but one had between (on average) 0.4 to 8.73 RT97 positive structures per section and the sizes of these structures varied from between 3.75 to $16.3 \mu \mathrm{m}$. Fewer stained axonal swellings were observed in the hippocampi of sham-lesioned and lesioned-only control rats and there was no obvious staining of perikarya in these control rats.

The RT97 antibody labels the phosphorylated $200 \mathrm{kDa}$ neurofilament protein found within axons in the normal CNS. Abnormal perikaryal staining with this antibody has been found in the brains of Alzheimer's patients where it labels neurofibrillary tangles $/ 1 /$ and following aluminium intoxication of the CNS /2/. Doering et al. $/ 3 /$ have observed perikaryal staining within 1 - 2 year old neural transplants and they also report one case with labelling of structures in the surrounding host tissue. Projection neurons whose axons have been severed also show an abnormal accumulation of phosphorylated neurofilaments (a phenomenon termed "the axon reaction") /4/. Here, RT97 staining of cell bodies and axonal swellings was found in the host hippocampus one year after intrahippocampal transplantation and therefore it is unlikely to be due to an axon reaction resulting from the lesioning of the fimbria-fornix. In addition, sham-lesioned and lesioned-only rats did not show any abnormal (clearly identified) perikaryal staining (only of axonal swellings). RT97 perikaryal staining was not found in neurons of the various hippocampal cell layers and may therefore be of interneurons. In three transplanted rats, there was also some damage to the medial CA1 cell layer and to a part of the molecular layer, which may have adversely affected the interneurons.

The selective damage to the corpus callosum, which was severe in one rat, is suggestive of 
diffuse axonal type injury which also leads to the formation of axonal retraction balls. An immunological response to myelin, akin to experimental allergic encephalomyelitis (EAE) in rats, may also have developed as a secondary consequence of the immunological rejection of the grafted tissue located on the cerebral cortex (Patel et al., submitted), which in these rats has led to necrosis of the cortex, abscess formation and ventricular enlargement.

Thus, under certain circumstances, some neuropathological consequences may develop following transplantation of neural allogeneic (or xenogeneic) tissue into the central nervous system. The immunological rejection mechanisms, although directed against the transplant, may cause damage to the host tissue, e.g. through the formation of abscesses, through antibody-mediated tissue injury and also through sustained phagocytic activity. In addition, prolonged and intense immunological rejection responses may also result in EAE type lesions and neural transplantation may also lead to abnormal changes within the surrounding host neurons, such as that shown by RT97 staining. Further long-term investigations of this sort need to be carried out in order to determine the causes of these changes.

\section{ACKNOWLEDGEMENTS}

We are grateful to the British Medical Research Council and the Wellcome Trust for financial support.

\section{REFERENCES}

1. Anderton BH et al. Nature $1982 ; 298: 84-86$.

2. Bizzi A et al. J Neurosci 1984; 4: 722-731.

3. Doering LC et al. Exp Neurol 1991; 111: 1-8.

4. Klosen P et al. Brain Res 1990; 526: 259-269.

5. Patel SN et al. Eur J Neurosci Suppl 1991; 4: 105. 

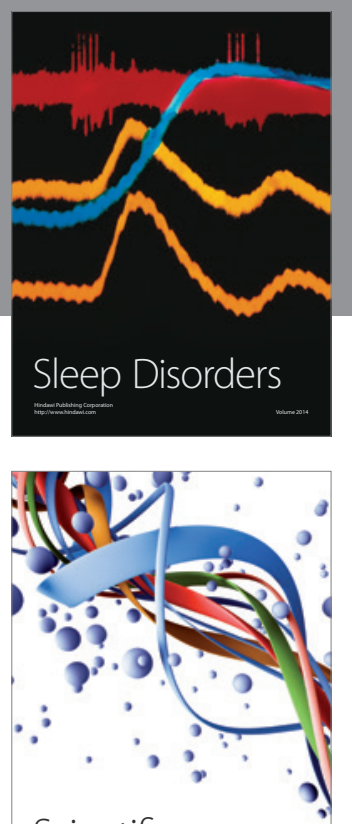

Scientifica
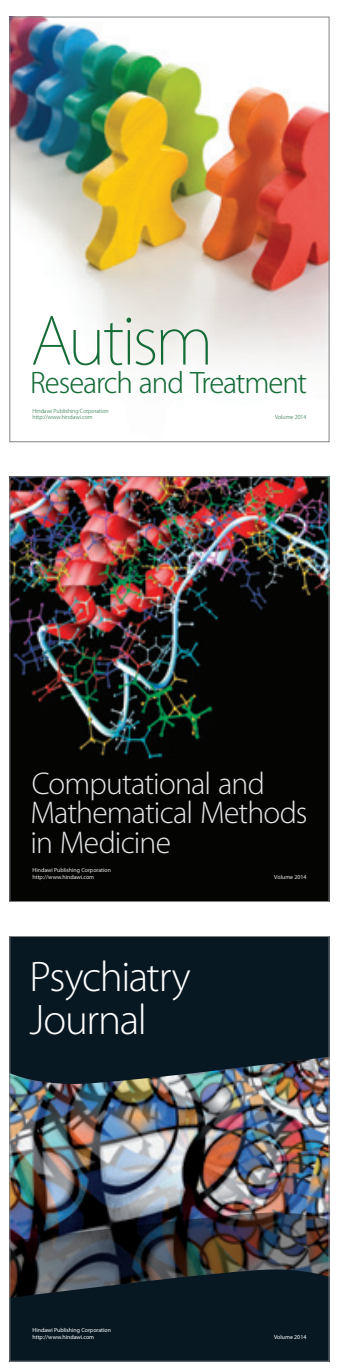
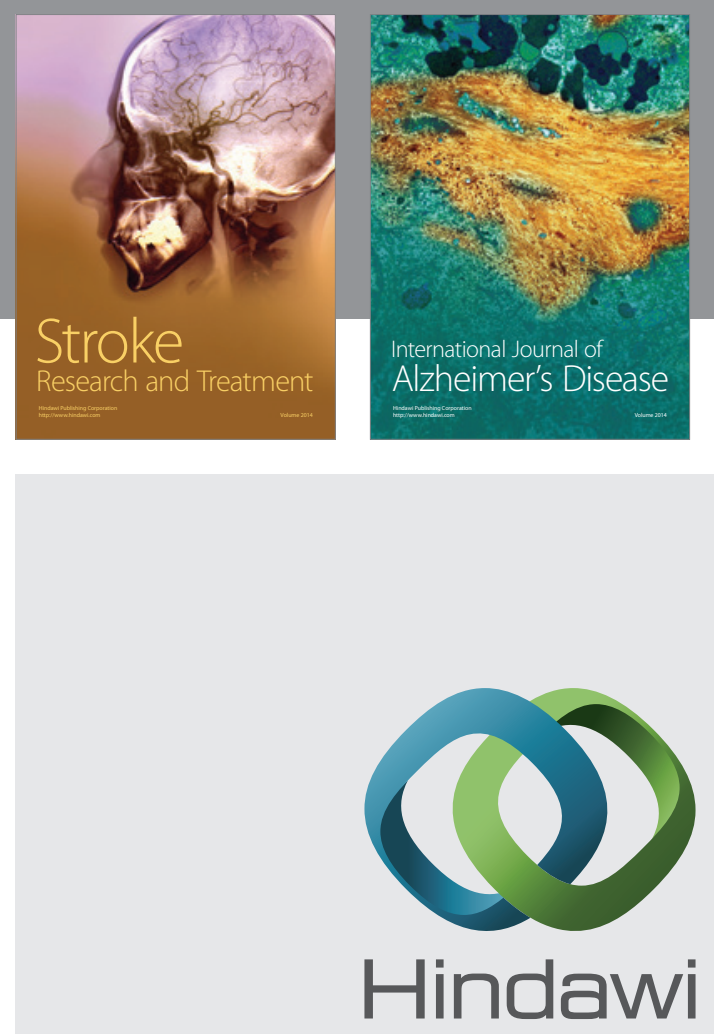

Submit your manuscripts at

http://www.hindawi.com
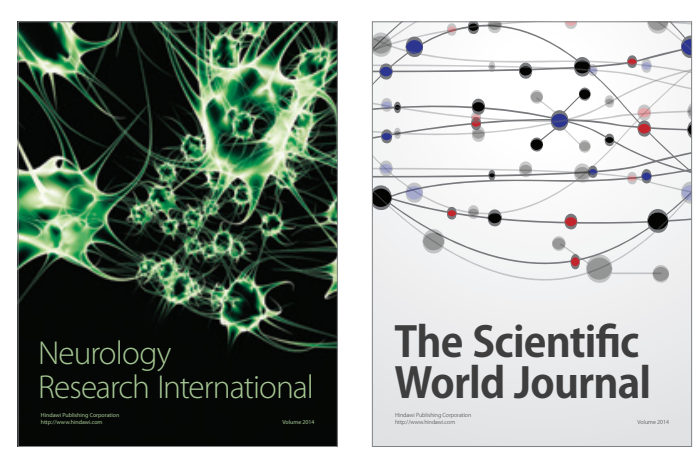

The Scientific World Journal

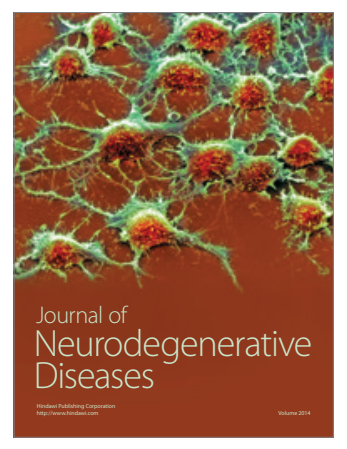

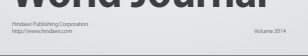

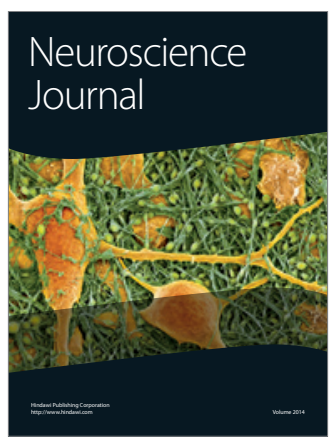

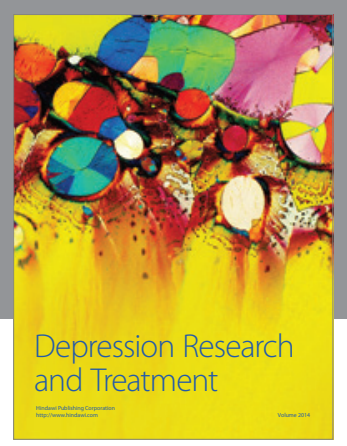
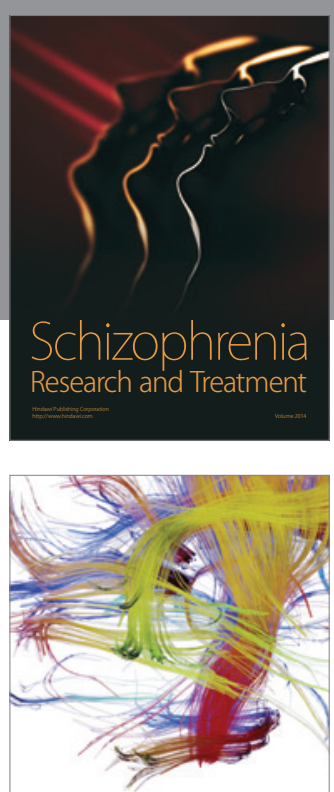

Brain Science

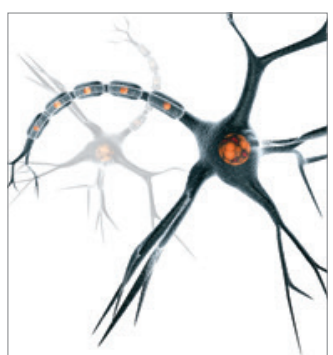

Neural Plasticity
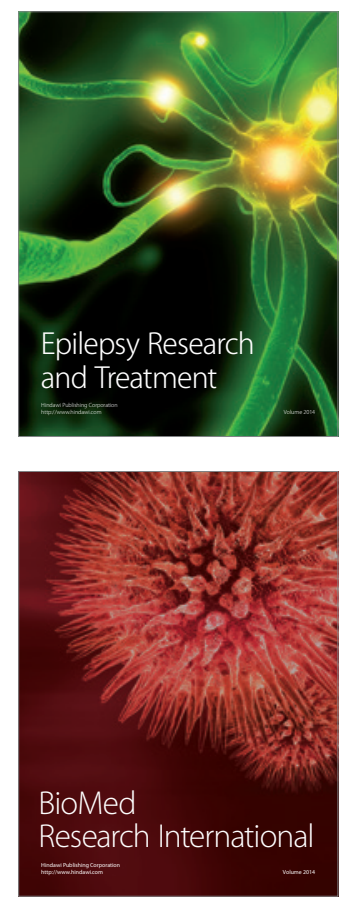

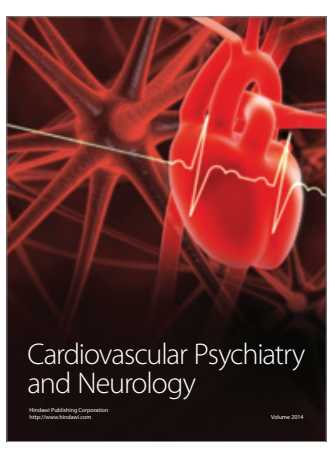

Parkinson's

Disease
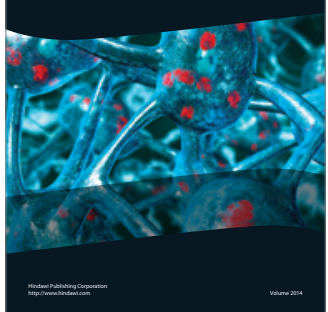